\title{
Analysis of Regional Left Ventricular Function in the Post-Infarct Mouse by Magnetic Resonance Imaging with Retrospective Gating
}

\author{
EG Caiani ${ }^{1}$, M Franzosi $^{1,2}$, L Castiglioni ${ }^{2}$, U Guerrini ${ }^{2}$ \\ ${ }^{1}$ Politecnico di Milano, Biomedical Engineering Dpt, Milan, Italy \\ ${ }^{2}$ Università degli Studi, Pharmacological Sciences Dpt, Milan, Italy
}

\begin{abstract}
Our aim was to test, in a murine myocardial infarction (MMI) model, the feasibility of a new MRI protocol with no ECG/respiratory gating, and compare normal mice versus MMI, in terms of global and regional $L V$ function. A control group (C, 10 mice) and a MMI group (18 mice) were studied. MRI with retrospective gating was performed. End-diastolic (EDV) and end-systolic (ESV) $L V$ volumes with ejection fraction (EF) were computed. In addition, regional fractional area change (RFAC) was used as index of regional wall motion. In MMI, LV size (in $\mu L$ ) was larger compared to $C$ (EDV: $92 \pm 25$ vs $36 \pm 4$,

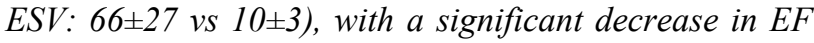
(31 \pm 10 vs $72 \pm 7 \%$ ). RFAC in $C$ showed heterogeneous values. In MMI, RFAC decreased from $L V$ base to apex, with a min (26\%) in the anterior segment, in agreement with the occluded LAD. MRI with retrospective gating is feasible in mice, and regional LV function analysis allows the localization of the induced infarction.
\end{abstract}

\section{Introduction}

Cardiovascular disease constitutes one of the leading causes of morbidity and mortality in industrialized countries [1]. Indeed, acute myocardial infarction represents the first cause of death $(30 \%)$ in the adult population of western countries.

To improve its prevention and treatment, in-vivo studies relying on animal models have become very important. In fact, they give new insights into the pathophysiology of myocardial infarction, and allow to experiment and test the effectiveness of new pharmacological therapies [2]. Murine myocardial infarction (MMI) models are used in the evaluation of new therapies for heart failure. However, results are limited by the unique aspects of mouse physiology, by the size of the animal and by the spatial and temporal resolution of the available imaging techniques.
In mice, magnetic resonance imaging (MRI) represents the method of choice for left ventricular (LV) volumetric quantification of cardiac function both for its high spatial and temporal resolution [3-5]. Through animal MRI equipment and workstation, it is possible to determine global cardiac functional indexes, such as end-diastolic volume (EDV), end-systolic volume (ESV), ejection fraction $(\mathrm{EF})$, stroke volume (SV) and ventricular mass [3-7]. However, the lack of regional LV functional analysis precludes the localization and quantification of regional wall motion defects. Moreover, the MRI technique is affected by cardiac and respiratory movements, which may generate artifacts in the images. Rhythm disturbances and respiratory variability may result, particularly in (MMI) models, in gating problems and long acquisition times, reducing data accuracy.

To date, the only way to deal with these problems is to synchronize the image sampling of the MRI scanner with the cardiac cycle (cardiac triggering) and to stop the image sampling during the respiratory period (respiratory gating). However, this procedure requires the duration of the examination to be increased, with a prolongation of the anesthesia for the mice, with relevant risks. [2]

We hypothesized that the utilization of a different acquisition protocol without triggering, and the availability of regional LV function information, could improve the reliability of results obtainable from MMI models.

Accordingly, our aims were: 1) to test the feasibility of a new cine MRI acquisition protocol with no ECG or respiratory gating; 2) to quantify both global and regional LV function from MRI murine images. To this respect, results from a mice control group will be compared with those from a group of MMI.

\section{Methods}

\subsection{Mouse preparation}

4 to 6 weeks female C57BL6 mice (body weight 20-25 
g) were studied. Mice were anesthetized with inhaled isofluorane (3-4 vol \% in 30/70 oxygen-nitrogen saturated chamber) and placed on a plexiglass plate, that was then introduced in the MR equipment. During the examination the isofluorane decreased to $1.5-2 \mathrm{vol} \%$, in order to maintain a deep anesthesia.

Ten mice constituted the control group (C).

In an additional group of 30 mice, a myocardial infarction was induced by ligation of the left anterior descending (LAD) coronary artery at mid LV level. To do so, mice were anesthetized with Domitor $16 \mu 1$ $(1 \mathrm{mmg} / \mathrm{Kg})$ e Ketavet (100) $32 \mu 1(75 \mathrm{mmg} / \mathrm{Kg})$ diluted in physiological solution. The trachea was intubated with a steel tube and ventilated with positive airway pressure (breathing volume of $140 \mu \mathrm{l}$ at $150 \mathrm{breaths} / \mathrm{min}$ ). [8] The left thoracotomy, with the dissection at the fourth intercostal space, allowed the exposition of the anterolateral heart surface. The myocardial infarction was induced by LAD ligation at the level of the bifurcation of the LAD with a 7.0 nylon thread. During the operation, the body temperature was maintained constant at $37.5^{\circ} \mathrm{C}$ with the use of an heating carpet. The operation took about 20-30 minutes; at the end, the thorax was closed and the animal was extubated and monitored. The Dormitor antidote, Antisedan, was administered at the same quantity of Dormitor to encourage the animal awakening.

In the $18 / 30$ mice surviving this operation, MRI was performed after 9 days (survival rate $60 \%$, comparable with [9]). These mice constituted the MMI group for comparison.

\subsection{In-vivo MRI}

Imaging was performed using a $4.7 \mathrm{~T}$ vertical-bore MR magnet (Bruker) using a gradient echo cine sequence with the following parameters: echo time $1.9 \mathrm{~ms}$; repetition time $10 \mathrm{~ms}$; field of view $4 \times 4 \mathrm{~cm}$; acquisition matrix $128 \times 128$ pixel; slice thickness $1.3 \mathrm{~mm}$. MR data acquisition was performed in multiple contiguous shortaxis slices, 12 frames for every cardiac cycle.

The images were acquired with new software (IntraGate, Bruker), based on a gradient echo sequence with a non spatially encoded navigator for every acquired echo. Phase and intensity of navigators are analyzed as a time dependent signal that provides information for retrospective reconstruction of high quality cardiac cine images, even if there are strong variations within the respiratory and/or cardiac rates. In this way, the use of ECG and respiration sensor instrumentation of the animal is avoided, because these parameter are evaluated retrospectively, and no triggering is required for image acquisition, thus shortening the examination time.

\subsection{Image analysis}

MRI Dicom images were imported in Matlab (The Mathworks Inc., Natick, MA) for analysis.

In each slice, endocardial border was semiautomatically detected frame-by-frame throughout the cardiac cycle. This was achieved by adaptive thresholding of videointensity based on pixel position. A binary image was created with a pixel position-dependent threshold [10], and further processed with standard morphological operators to extract the LV cavity. Thresholding parameters were initially adjusted for the first frame in each image sequence by visually verifying the position of the resulting contour, which was superimposed on the original image. Then, the optimal parameters were applied to the subsequent images, and adjusted when necessary to optimize border position for that particular frame. LV cavity area was then calculated frame-by-frame directly from pixel counts inside the detected endocardial border.

To obtain global indices, for each frame, LV volumes were derived by summing the LV cavity areas obtained for each slice, and multiplying it by the slice thickness. From these LV volume versus time curves, the endsystolic (ESV) and end-diastolic volumes (EDV) were defined as minimum and maximum in the cardiac cycle, from which stroke volume (SV) was derived, and ejection fraction (EF) was computed as $100 * \mathrm{SV} / \mathrm{EDV}$. Also, from the first time derivative, peak filling (PFR) and peak ejection (PER) rates were calculated, as absolute maximum and minimum, respectively.

To obtain regional indices, for every slice, the LV cavity centroid was automatically calculated in the ES frame, and used as the origin of segmentation. An additional point was then manually placed at the junction between the right ventricular free wall and the interventricular septum. Starting from that point, the LV cavity was automatically divided into six $60^{\circ}$ wedgeshaped sectors. For each segment, regional fractional area change (RFAC) in \% of regional end diastolic area was calculated automatically and used as index of regional wall motion [10].

To allow averaging of RFAC in mice with different number of slices covering the LV from base to apex, values from different slices in each mouse were resampled using cubic spline interpolation to obtain 100 values from base to apex for each sector in which the LV wall was divided (anterior, anteroseptal, septal, lateral, posterior and inferior).

For all mice in the two considered groups $(\mathrm{C}$ and MMI), these values were then averaged on a point-bypoint basis to obtain mean RFAC and the corresponding $\mathrm{SD}$, every $1 \%$ of the LV length from base to apex. 


\section{Results}

Of the 30 mice undergoing LAD ligation, 11 died due to complicances during surgery or post-surgery, while 1 died during the MR acquisition.

Using the new software for retrospective gating, the time needed to acquire a single slice was reduced from $6 \sim 8$ minutes of the traditional acquisition to $4 \sim 5$ minutes. The results for the global parameters obtained in the two groups of mice are shown in table 1. As expected, all parameters except SV showed significant differences.

Table 1. Comparison of EDV,ESV,SV,EF,PFR and PER between the control group (C) and the infarcted (MMI) mice (unpaired t-test, *: $\mathrm{p}<.05 \mathrm{C}$ vs MMI).

\begin{tabular}{ccc} 
Parameter & C & MMI \\
\hline EDV $(\mu \mathrm{L})$ & $36 \pm 4$ & $92 \pm 25^{*}$ \\
ESV $(\mu \mathrm{L})$ & $10 \pm 3$ & $66 \pm 27^{*}$ \\
SV $(\mu \mathrm{L})$ & $26 \pm 3$ & $26 \pm 4$ \\
EF $\%$ & $72 \pm 7$ & $31 \pm 10^{*}$ \\
PFR (u.a.) & $3.6 \pm 4$ & $1.9 \pm 5^{*}$ \\
PER (u.a.) & $-3 \pm 0.5$ & $-1.8 \pm 0.8^{*}$ \\
\hline
\end{tabular}

Figure 1 shows the results of mean RFAC computed for every $1 \%$ of the LV length, from base to apex, for the $\mathrm{C}$ group. It is worth noting that every segment resulted in high RFAC values, with some decrements in the anteroseptal and septal sectors at base levels.

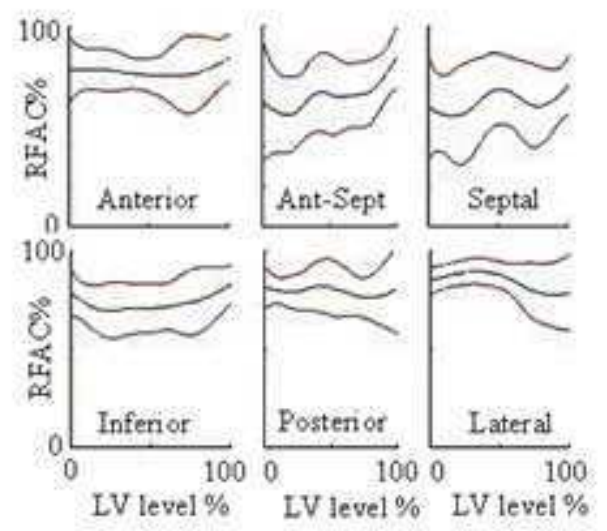

Figure 1. Mean $\mathrm{RFAC} \pm \mathrm{SD}$ representation from base (0) to apex (100) of the LV length for the $\mathrm{C}$ group in all circular cardiac segments.

To allow an easy visualization and comparison of the regional results obtained from the $\mathrm{C}$ and $\mathrm{MMI}$ groups, for each sector the mean RFAC was resampled every $10 \%$, and displayed in a "bull's eye" format, where darker colors represent abnormal segments (Figure 2). It appears evident how the MMI group had lower RFAC values compared to the $\mathrm{C}$ group, in particular for mid and apical $\mathrm{LV}$, with anterior and anteroseptal segments primarily affected by the infarction.
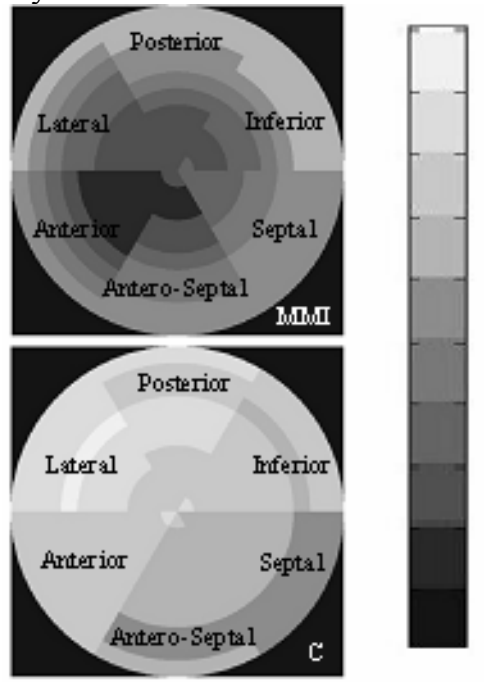

100

RFAC

0

Figure 2. Bull's eye representation of color encoded RFAC for each cardiac sector between mice with MI (top) and control group (bottom). The inner circle represents the LV apex, while the outer circles represent the other slices from the LV apex to base. Light color shows normal LV function, while darker colors reflect reduced regional $\mathrm{LV}$ function.

In table 2, the mean RFAC values for each sector, averaged form base to apex, obtained from $\mathrm{C}$ and MMI groups, are shown.

Table 2. Representation of mean RFAC for each cardiac sector in control group and in MI mice

\begin{tabular}{ccc} 
LV sector & C & MMI \\
\hline Anterior & $77 \pm 12$ & $26 \pm 11$ \\
Anteroseptal & $65 \pm 18$ & $34 \pm 12$ \\
Septal & $62 \pm 20$ & $43 \pm 15$ \\
Inferior & $73 \pm 13$ & $45 \pm 17$ \\
Posterior & $79 \pm 12$ & $42 \pm 19$ \\
Lateral & $84 \pm 10$ & $35 \pm 15$ \\
\hline
\end{tabular}

\section{Discussion and conclusions}

Cine MRI with retrospective gating in mice was feasible, reducing acquisition time, thus shortening the anaesthesia with lower risk for the animal. An additional benefit of this procedure is represented by the fact that it is free from problems due to electric ECG wire interference, to the erroneous detection of ECG R-wave, in particular in animals with myocardial infarction, or to strong respiratory and cardiac rates variations during MR acquisition. 
In MMI group, at 9 days form the LAD ligation, LV cavity resulted dilated, with reduced EF, PER and PFR, due to cardiac remodeling. [11]. On the contrary, the SV in the two groups was comparable, in coherence with the same level of cardiac output. These results are in agreement with previous values reported in literature for similar studies [12-16]. Computation of regional ventricular function parameters (RFAC) allowed the localization of the effects of the induced infarction in the MMI group. In the $\mathrm{C}$ group, RFAC showed heterogeneous values in the different sectors, with max and $\min$ in the lateral (84\%) and septal (62\%) segments, respectively. In MMI, RFAC decreased in all sectors, with a min $(26 \%)$ in the anterior segment. A progressive $\mathrm{RFAC}$ reduction is also noticeable from base to apex.

From the bull's eye representation, it is possible to visualize the effects of the LAD occlusion in every sector and slice, with a general reduction of the RFAC at the apical and mid LV level. In the control group, a lower values of RFAC were visible in the septal and anteroseptal sectors, probably due to the effects of ventricular interdependence.

In conclusion, in mice studies cine MRI with no ECG and respiration triggering and retrospective gating is feasible, reducing acquisition time and trigger-related problems. Regional LV function analysis allows the localization of the induced infarction, with potential applications in clinical trials on small animals to assess the localized effectiveness of pharmacological treatments or regenerative medicine.

\section{References}

[1] Levy RI, Moskowitz J. Cardiovascular research: decades of progress, a decade of promise. Science 1982;217:121-9.

[2] Monassier L, Constantinesco A. Cardiovascular disorders: insights into in-vivo cardiovascular phenotyping. In: Standards of mouse model phenotyping. Editors: De Agelis MH, Chambon P, Brown S. Wiley VCH, Weinheim 2006;7:177-99.

[3] Ruff J, Wiesmann F, Hiller KH, Voll S, von Kienlin M, Bauer WR, Rommel E, Neubauer S, Haase A. Magnetic resonance microimaging for noninvasive quantification of myocardial function and mass in the mouse. Magn Reson Med 1998;40:43-8.

[4] Weiss RG, Chacko SM, Aresta F, Chacko VP. Dynamic magnetic resonance images of murine cardiac function at physiological heart rates. Circulation 2000;101:e92.

[5] Wiesmann F, Ruff J, Hiller KH, Rommel E, Haase A, Neubauer S. Developmental changes of cardiac function and mass assessed with MRI in neonatal, juvenile, and adult mice. Am J Physiol Heart Circ Physiol 2000;278:H652-7.

[6] Semelka RC, Tomei E, Wagner S,Mayo J et al. Interstudy reproducibility of dimensional and functional measurements between cine magnetic resonance studies in the morphologically abnormal left ventricle. Am Heart J
1990;119:1367-73.

[7] Florentine MS, Grosskreutz CL, Chang W, Hartnett JA, Dunn VD, Ehrhardt JC, Fleagle SR, Collins SM, Marcus ML, Skorton DJ. Measurement of left ventricular mass in vivo using gated nuclear magnetic resonance imaging. J Am Coll Cardiol 1986;8:107-12.

[8] Tarnawski O., McMullen J.R., Schinke M., Nie Q., Kong S., Izumo S. Mouse cardiac surgery: comprehensive techniques for the generation of mouse models of human deseases and their application for genomic studies. Physiological Genomic 2004;16:349-60.

[9] Kumar D., Hacker T., Buck J., Whitessel L.F., Keji E.H., Douglas P.S., Kamp T.J. Distinct mouse coronary and myocardial infarction consequence to ligation. Pathophysiology and natural history 2005;16(1):41-4.

[10] Caiani EG, Toledo E, MacEneaney P, Bardo D, Cerutti S, Lang RM, Mor-Avi V. Automated interpretation of regional left ventricular wall motion from cardiac magnetic resonance images. J Cardiovasc Magn Res 2006;8:427-33.

[11] Sutton MJ, Sharpe N. Left ventricular remodeling after myocardial infarction. Circulation 2000;101: 2981-8.

[12] Dawson D, Lygate CA, Saunders J, Schneider JE, Ye X, Hulbert K, Noble JA, Neubauer S. Quantitative 3dimensional echocardiography for accurate and rapid cardiac phenotype characterization in mice. Circulation 2004;110(12):1632-7.

[13] De Celle T, Cleutjens JP, Blankesteijn WM, Debets JJ, Smits JF, Janssen BJ. Long term strumental and functional consequences of cardiac ischaemia-reperfusion injury in vivo mice. Experimental Physiology 2004;89(5):605-15.

[14] Nahrendorf M, Badea C, Hedlund LW, Figueiredo JL, Sosnovik DE, Johnson GA, Weissleder R. High resolution imaging of murine myocardial infarction with delayed enhancement Cine Micro-CT. Am J Physiol Heart Circ Physiol; 2007;292:3172-8.

[15] Schneider JE, Cassidy PJ, Lygate C, Tyler DJ, Wiesmann F, Grieve SM, Hulbert K, Clarke K, Neubauer S. Fast, high-resolution in vivo cine magnetic resonance imaging in normal and failing mouse hearts on a vertical $11.7 \mathrm{~T}$ system. J Magn Res Imaging 2003;18:691-701.

[16] Wiesmann F, Ruff J, Engelkardt S, et al. Dobutaminestress magnetic resonance microimaging in mice: acute changes of cardiac geometry and function in normal and failing murine hearts. Circulation Res 2001;88:563-9.

Address for correspondence

Enrico Caiani, $\mathrm{PhD}$

Dipartimento di Bioingegneria - Politecnico di Milano

Piazza L. da Vinci 32, Milano, Italy

E-mail: caiani@biomed.polimi.it 